\title{
The origin of slow electron recombination processes in dye-sensitized solar cells with alumina barrier coatings
}

\author{
Francisco Fabregat-Santiago and Jorge García-Cañadas \\ Departament de Ciències Experimentals, Universitat Jaume I, 12080 Castelló, Spain \\ Emilio Palomares \\ Department of Chemistry, Imperial College, London SW7 2AY, United Kingdom and Institut de Ciència \\ Molecular Universitat de València, C. P. 46100, Dr. Moliner 50, Burjassot, València, Spain \\ John N. Clifford, Saif A. Haque, and James R. Durrant \\ Department of Chemistry, Imperial College, London SW7 2AY, United Kingdom
}

Germà Garcia-Belmonte and Juan Bisquert ${ }^{\mathrm{a})}$

Departament de Ciències Experimentals, Universitat Jaume I, 12080 Castelló, Spain

(Received 14 June 2004; accepted 9 September 2004)

\begin{abstract}
We investigate the effect of a thin alumina coating of nanocrystalline $\mathrm{TiO}_{2}$ films on recombination dynamics of dye-sensitized solar cells. Both coated and uncoated cells were measured by a combination of techniques: transient absorption spectroscopy, electrochemical impedance spectroscopy, and open-circuit voltage decay. It is found that the alumina barrier reduces the recombination of photoinjected electrons to both dye cations and the oxidized redox couple. It is proposed that this observed retardation can be attributed primarily to two effects: almost complete passivation of surface trap states in $\mathrm{TiO}_{2}$ that are able to inject electrons to acceptor species, and slowing down by a factor of 3-4 the rate of interfacial charge transfer from conduction-band states. () 2004 American Institute of Physics. [DOI: 10.1063/1.1812588]
\end{abstract}

\section{INTRODUCTION}

New concepts for photovoltaic energy conversion are increasingly focusing at the intersection between organic and inorganic materials. Such devices are based upon nanometerscale interpenetrating networks of electron and hole transporting materials ${ }^{1-3}$ rather than employing the long diffusion length, high-purity crystals, as used in conventional silicon solar cells. Dye-sensitized solar cells (DSSCs) are so far the most efficient devices based on this concept. DSSCs base their ability to convert photon energy to electricity on fast conversion of photogenerated excitons to energetic charge carriers spatially separated in distinct nanoscale domains or phases. The energy associated with photoexcitation of the sensitizer dye is employed to inject electrons into the conduction band of nanocrystalline $\mathrm{TiO}_{2}$ and the holes into the redox electrolyte, generally the $I^{-} / I_{3}^{-}$redox couple in an organic solvent. Under illumination, the Fermi level of electrons in the $\mathrm{TiO}_{2}$ is raised ${ }^{4}$ relative to the chemical potential of the redox couple in the electrolyte, resulting in a large thermodynamic driving force (ca. $0.8 \mathrm{eV}$ ) for electron transfer from the $\mathrm{TiO}_{2}$ to the redox couple. Slow kinetics for this charge recombination process are therefore essential to allow the charge carriers enough time to be collected into their respective contacts. ${ }^{5}$

Recently, the concept of a "kinetic barrier" for interfacial charge recombination processes in DSSCs has been further demonstrated by the use of ultrathin $(\leqslant 1 \mathrm{~nm})$ conformal alumina $\left(\mathrm{Al}_{2} \mathrm{O}_{3}\right)$ layers deposited in situ onto the surface on the nanocrystalline metal oxide films, leading to a significant

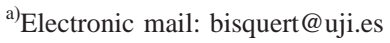

increase in DSSC energy conversion efficiencies, as reported by several groups. ${ }^{6-12}$ Palomares et $a l .{ }^{11}$ investigated the electron recombination process at the interface between $\mathrm{TiO}_{2}$ and the oxidized dye, and have shown slow kinetics for the electron transfer process when the $\mathrm{TiO}_{2}$ nanocrystalline particles were coated with $\mathrm{Al}_{2} \mathrm{O}_{3}$. However, direct evidence for slow recombination kinetics between the electrons in the $\mathrm{TiO}_{2}$ and the oxidized species in the electrolyte $\left(I_{3}^{-}\right)$were not addressed. Due to the strong interest in this approach, ${ }^{12,13}$ we decided to investigate further the origin of the efficiency improvement induced by the $\mathrm{Al}_{2} \mathrm{O}_{3}$ coating. Our study combines the interpretation of several experimental techniques which address the charge-transfer processes between electrons in the $\mathrm{TiO}_{2}$ and either the oxidized dye or the oxidized species $\left(I_{3}^{-}\right)$in the electrolyte. First, micro-millisecond transient absorption spectroscopy is employed to give detailed information on interfacial electron transfer kinetics at the dye-sensitized photoelectrode as a function of the applied potential in the absence of the $I_{3}^{-} / I^{-}$redox couple, as discussed previously. ${ }^{11}$ In this study we complement this technique with studies of complete DSSCs employing both electrochemical impedance spectroscopy (EIS), a technique that has been applied to nanostructured semiconductor electrodes, ${ }^{14-16}$ and the more recently developed ${ }^{17}$ opencircuit voltage decay (OCVD) analysis. The OCVD analysis consists of interrupting a steady-state illumination of devices at open circuit and monitoring the subsequent decay of photovoltage (PV), $V_{o c}$. These complementary techniques allow us to report measurements of electron lifetimes by independent methodologies, providing macroscopic parameters at 
different steady states in the operation conditions of the DSSC.

The modulation of voltage imposed by the external source in the EIS technique induces a variation of the Fermi level of the nanocrystalline $\mathrm{TiO}_{2}$ electrode. This has two effects: (i) it changes the occupancy of electronic states of electrode and (ii) it promotes a recombination current across the interface. In the EIS experiment, effect (i) corresponds to the chemical capacitance $C_{\mu}$, as discussed in previous works, ${ }^{18,19}$ and (ii) corresponds to a charge-transfer (recombination) resistance $R_{c t}$. The product of these quantities defines a time constant, $\tau_{n}=R_{c t} C_{\mu}$, which corresponds to the observed electron lifetime. However, this lifetime contains components that may be associated both with interfacial charge recombination dynamics and also with the internal steps of charge reorganization in the semiconductor energy levels (conduction-band and band-gap states). Therefore, $\tau_{n}$ $=R_{c t} C_{\mu}$ as determined from the EIS data relates more properly to the response time that has the general meaning of the characteristic time for the decay following a small perturbation. ${ }^{18,20}$ In contrast, in the open-circuit voltage decay measurements, the photovoltage decays without an external influence, and the response time is obtained by the reciprocal of the derivative of the decay curve normalized by the thermal voltage,

$$
\tau_{n}=-\frac{k_{B} T}{e}\left(\frac{d V_{o c}}{d t}\right)^{-1} .
$$

This technique has the advantage of providing detailed resolution of the electron lifetime over a wide range of variation of the Fermi level ${ }^{17}$ and provides an alternative measurement of the electron lifetime complementary to EIS.

\section{EXPERIMENTAL SECTION}

The DSSCs were prepared employing both uncoated $\left(\mathrm{TiO}_{2}\right)$ and coated $\left(\mathrm{TiO}_{2} / \mathrm{Al}_{2} \mathrm{O}_{3}\right)$ nanocrystalline films (film thickness is $4 \mu \mathrm{m}$ ) on TEC15 $\mathrm{SnO}_{2}: \mathrm{F}$ glass and the sensitizer dye $\mathrm{Ru}(\mathrm{dcbpy})_{2}(\mathrm{NCS})_{2}$, as reported previously. ${ }^{11}$ The electrolyte comprised 0.6-M tetrabutyl ammonium iodide, 0.1-M LiI, 0.1-M I $\mathrm{I}_{2}$, and 0.5-M 4-tert-butyl pyridine in anhydrous acetonitrile. All the DSSCs used had a $0.9-\mathrm{cm}^{2}$ geometric area.

The transient absorption experiments have been described elsewhere, employing sensitized electrodes immersed in a redox inactive electrolyte in a three-electrode photoelectrochemical cell. ${ }^{11}$ In the impedance measurements, the DSSCs were illuminated with a 150-W Xe Lamp (Oriel Instruments). An ultraviolet (UV) filter was used to decrease the UV component from the light beam. Several light absorber filters were used as well to get the desired light intensity. Light intensity was measured with a quantum photo radiometer (Delta Ohm HD 9021). The open-circuit voltage decay measurements for $\mathrm{TiO}_{2} / \mathrm{Al}_{2} \mathrm{O}_{3}$ and $\mathrm{TiO}_{2}$ solar cells were performed by monitoring the photovoltage decay following interruption of the stationary illumination in the same conditions as EIS. The decays were recorded using an Ecochemie potentiostat equipped with a short interval sampling module. Typically the measurement interval was $10-50 \mathrm{~ms}$.

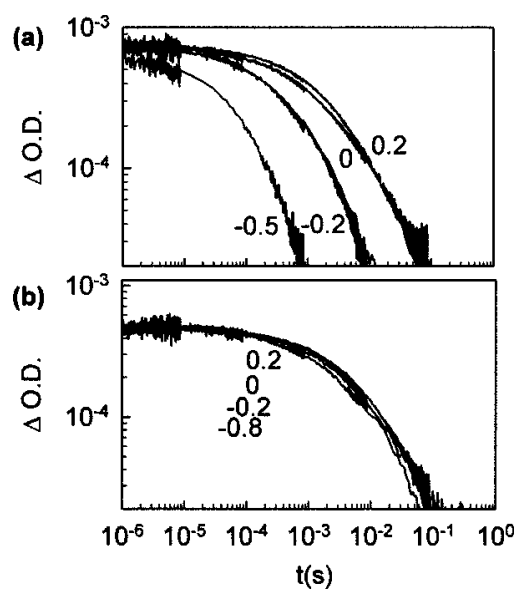

FIG. 1. Transient absorption data monitoring the photoinduced absorption of the dye cation following optical excitation of the dye $\mathrm{RyL}_{2}(\mathrm{NCS})_{2}$ adsorbed on nanocrystalline $\mathrm{TiO}_{2}$ films without (a) and with (b) $\mathrm{Al}_{2} \mathrm{O}_{3}$ overlayer coating, at different bias potentials shown in Volts $\mathrm{V}$.

\section{RESULTS AND DISCUSSION}

The transient absorption measurements illustrated in Fig. 1 monitor the reduction of dye cations generated by pulsed laser excitation of the dye-sensitized film in a three-electrode photoelectrochemical cell. The bias potential controls the electrode Fermi level relative to the reference electrode and thereby the average number of electrons present in the semiconductor. The decays for the uncoated $\mathrm{TiO}_{2}$ electrode, Fig. 1(a), show that application of a negative bias considerably accelerates the kinetics of dye cation rereduction. ${ }^{21,22}$ It was found that the bias dependence of half decay times is consistent with an exponential distribution of traps in $\mathrm{TiO}_{2}{ }^{22,23}$ Figure 1(b) shows that the accelerating effect of the negative bias is almost completely suppressed by the $\mathrm{Al}_{2} \mathrm{O}_{3}$ barrier coating. In the limit of positive applied potential, corresponding to conditions where the electron density in the film is dominated by electrons injected by the laser pulse, the recombination half-time for the coated film is longer than for the uncoated film, indicating that the $\mathrm{Al}_{2} \mathrm{O}_{3}$ provides a kinetic barrier to charge recombination to dye cations, as we have discussed previously. ${ }^{11}$

We have previously found that retardation of the recombination dynamics is most effective with barrier coating with high points of zero charge (basic coatings) and correlated this suppression with shifts in the conduction/trap density of states (DOS) attributed to the acid/base nature of the coating. ${ }^{11}$ However, we note that this argument is not sufficient to explain quantitatively the almost complete suppression of the bias dependence shown in Fig. 1(b). Chronoamperometric studies of electron density indicate that over this bias range the electron density in the $\mathrm{Al}_{2} \mathrm{O}_{3}$-coated film still increases by two orders of magnitude. ${ }^{11} \mathrm{We}$ can therefore conclude that for the $\mathrm{Al}_{2} \mathrm{O}_{3}$-coated films, the kinetics of recombination are practically independent of the number of electrons fixed in the $\mathrm{TiO}_{2}$ by the bias potential. This conclusion suggests that the recombination kinetics for the $\mathrm{Al}_{2} \mathrm{O}_{3}$-coated films are strongly controlled by interfacial charge-transfer dynamics rather than electron trapping/ detrapping dynamics within the $\mathrm{TiO}_{2}$ electrode. The release 


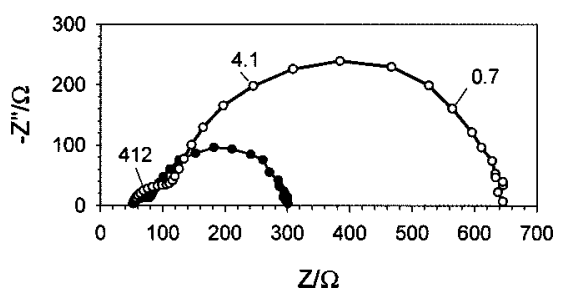

FIG. 2. Impedance spectra of illuminated dye-sensitized solar cells of $\mathrm{Al}_{2} \mathrm{O}_{3}$-coated nanocrystalline $\mathrm{TiO}_{2}$ at different steady states under galvanostatic (open circuit) conditions. Illumination at 0.1 sun $(\bigcirc)$ and 0.05 sun $(\bullet)$ (Xe lamp). Frequency values are indicated in $\mathrm{s}^{-1}$.

times for the trapped electrons in the $\mathrm{TiO}_{2}$ depends exponentially on the energy difference between the highest-filled electron trap and the conduction-band edge. We have previously shown that such detrapping dynamics play a key role in determining the recombination dynamics of dye-sensitized $\mathrm{TiO}_{2}$ films in the absence of $\mathrm{Al}_{2} \mathrm{O}_{3}$ coatings, ${ }^{21-23}$ resulting in, for example, the strong bias dependence of the recombination dynamics observed for the uncoated film in Fig. 1(a). The absence of any significant bias dependence for the recombination dynamics of the coated film suggests that such detrapping dynamics do not significantly influence the recombination dynamics of the coated film, but rather that interfacial kinetics are much slower and govern the transient response. In general, interfacial charge transfer may occur through a distribution of trap/surface states, ${ }^{24}$ but in this case one expects the kinetics to be strongly bias dependent because at higher values of stationary Fermi level, an increasing number of trap/surface states are occupied with electrons and participate in the charge transfer. This is identified in Ref. 23 as the main reason for the dependence of recombination kinetics upon negative bias. In addition, assuming that the charge transfer to the oxidized dye is isoenergetic, as demonstrated recently for $I_{3}^{-}$acceptor, ${ }^{25}$ it is important to consider the match of trap/surface states in the semiconductor with the fluctuating states of the oxidized dye (Gaussian distribution of half width $\lambda$ ), ${ }^{26}$ especially taking into account the rather large reorganization energies $\lambda \approx 0.8-1.2 \mathrm{eV}$ that have been reported for the oxidized dyes in DSSC. ${ }^{27}$ Thus one can understand the behavior observed in Fig. 1(a) as an increasing rate of recombination due to the increase with the negative bias of both density of occupied trap/surface states and available number of acceptor energy levels. In contrast, these effects are completely absent for the alumina-coated $\mathrm{TiO}_{2}$ in Fig. 1(b). The suggested explanation of the different behaviors is shown in Scheme 1. We suggest that the results of Fig. 1 indicate that the alumina covering produces a strong suppression of the kinetic effect of charge transfer from surface trap states in the $\mathrm{TiO}_{2}$ particles, as we will discuss in more detail below.

Of particular relevance to the above discussion, Dittrich et $a{ }^{28}{ }^{28}$ have recently demonstrated that the deposition of ultrathin alumina layers produces a strong passivation of surface states of $\mathrm{TiO}_{2}$ films. This study employed transient PV spectroscopy to show that the PV signal increases already at photon energies above $1 \mathrm{eV}$ for the uncoated $\mathrm{TiO}_{2}$, indicating strong absorption at defect states, while the $\mathrm{TiO}_{2} / \mathrm{Al}_{2} \mathrm{O}_{3}$ films show PV signal only above $3.1 \mathrm{eV}$, the band-gap of
$\mathrm{TiO}_{2}$. The authors suggested that the ultrathin alumina layer prevented the formation of defects by stabilizing the chemical potential of oxygen at the $\mathrm{TiO}_{2}$ surface, ${ }^{28}$ in good agreement with our hypothesis detailed above.
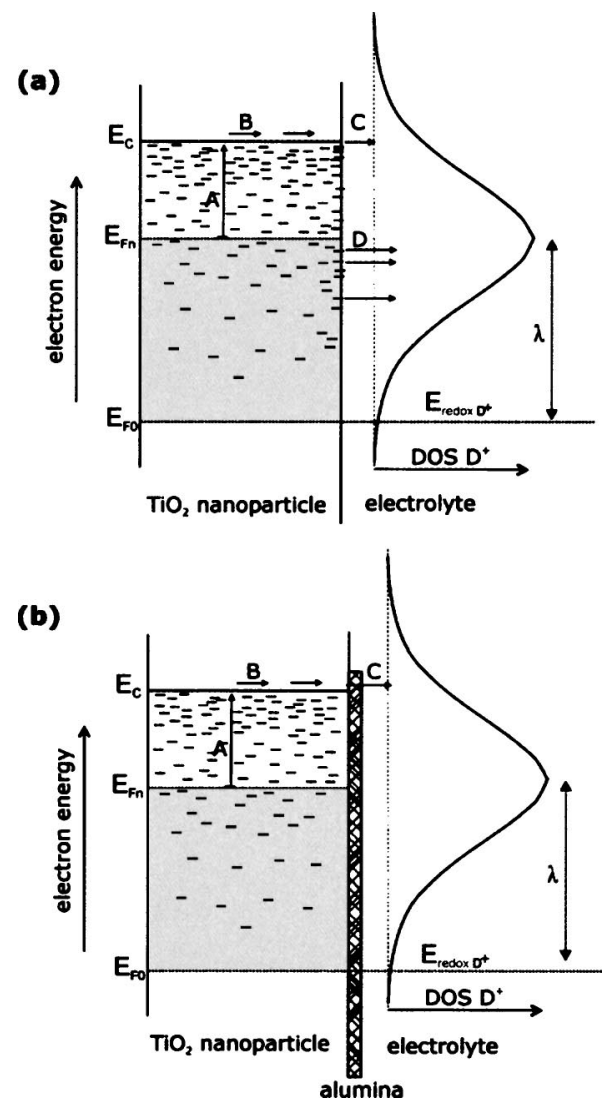

SCHEME 1. Schematics of the steps involved in the reduction of oxidized dye molecules by electron transfer from $\mathrm{TiO}_{2}$ semiconductor nanoparticles for (a) bare and (b) ultrathin alumina-coated $\mathrm{TiO}_{2} .\left(E_{F 0}\right)$ shows the position of the Fermi level in the dark, which is equilibrated with the redox potential $\left(E_{\text {redox }} \mathrm{D}^{+}\right)$of the oxidized dye. $\left(E_{F n}\right)$ is the quasi-Fermi level of electrons under a negative bias potential and $E_{C}$ is the conduction-band energy. The shaded region indicates the band gap states that are occupied with electrons. The following steps are indicated: (A) thermal release from an exponential distribution of band-gap localized states; (B) electron transport through extended states; (C) electron transfer through conduction band to the fluctuating energy levels of oxidized dye $D^{+}$in solution, which form a distribution with an effective Gaussian DOS, indicated in the right, with half width $\lambda$, the reorganization energy; (D) charge transfer from occupied surface states to the acceptor states at the same energy level. In (b), it is indicated that the alumina coating passivates the band-gap states close to the particle surface, leaving the transfer from conduction band $(\mathrm{C})$ as the only transfer mechanism.

Figure 2 shows the characteristic electrochemical impedance spectra for DSSC, consisting of two semicircles. The one at high frequencies is attributed to the counter electrode charge transfer, ${ }^{16}$ and the larger one at low-frequencies is attributed to electron accumulation and recombination processes in the nanocrystalline film. The steady state was fixed by imposing zero current at a given illumination intensity of the solar cell. In practice, the cell is set at an open-circuit condition by this procedure. The low frequency semicircle was fitted to a charge-transfer resistance $R_{c t}$ in parallel with the chemical capacitance $C_{\mu}$ and both of them in series with a resistance $R_{s}$. The resulting parameters for both different devices are shown in Fig. 3.

The lifetime (response time), $\tau_{n}=R_{c t} C_{\mu}$, determined 
(a)

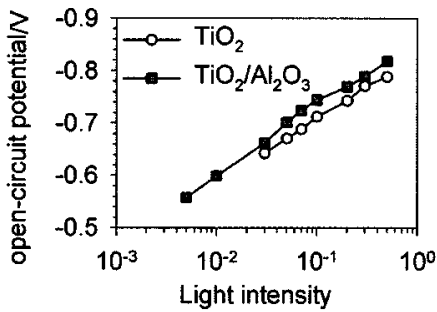

(b)

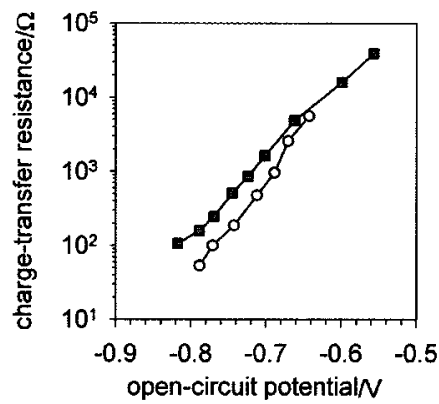

(c)

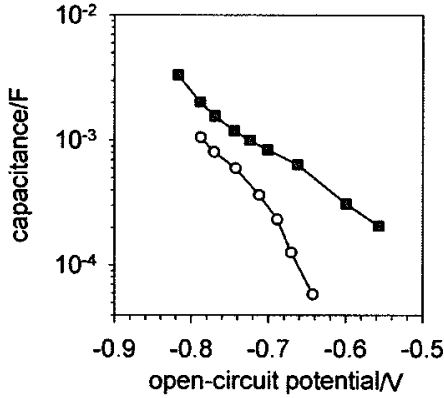

(d)

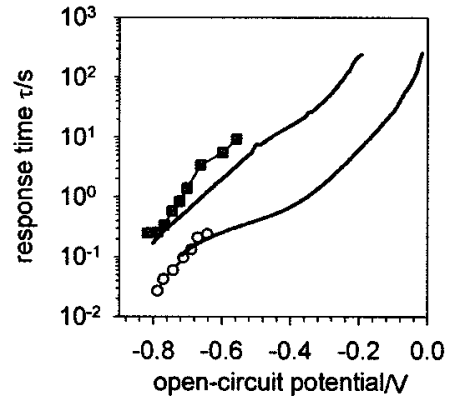

FIG. 3. Parameters resulting from electrochemical impedance spectroscopy measurements of illuminated nanocrystalline dye-sensitized solar cells with uncoated $\mathrm{TiO}_{2}$ and $\mathrm{Al}_{2} \mathrm{O}_{3}$-coated $\mathrm{TiO}_{2}$ at varying steady states under galvanostatic (zero current, open circuit) conditions. (a) open-circuit potential with respect to illumination intensity of Xe lamp in suns, (b) charge-transfer resistance $R_{c t}$, (c) film (chemical) capacitance $C_{\mu}$, and (d) (data points) response times, $\tau_{n}=1 / R_{c t} C_{\mu}$ determined from the EIS data. Also shown in (d) (bold lines) are response times determined by open-circuit photovoltage decays for the uncoated and coated DSSCs.

from the EIS data shown in Figs. 3(b) and 3(c) is shown in Fig. 3(d) for $\mathrm{TiO}_{2}$ and $\mathrm{TiO}_{2} / \mathrm{Al}_{2} \mathrm{O}_{3}$. The $\mathrm{TiO}_{2} / \mathrm{Al}_{2} \mathrm{O}_{3}$ curve shows a relative increase of the lifetime by about one order of magnitude when compared with $\mathrm{TiO}_{2}$. As mentioned above, the response time describes not only the transfer kinetics but also the reorganization of electrons by diffusion in energy space in the internal energy levels of the semiconductor particles. ${ }^{18}$ However, this effect influences the response time only through $C_{\mu}$, while $R_{c t}$ relates exclusively to the interfacial charge-transfer events. The lifetime data determined from the EIS data, as shown in Fig. 3(d), are restricted to high photovoltages and are therefore most likely describing the conditions in which the mechanism of charge transfer determining $R_{c t}$ is the direct transfer from the $\mathrm{TiO}_{2}$ conduction band. The approximately exponential dependence of the lifetime on the Fermi level seen in the impedance results of Fig. 1(d) can be interpreted as a result of trapping and release of electrons between the internal traps and the conduction band. This model ${ }^{17,18}$ indeed predicts an exact exponential dependence. A key point is that the relative increase of the lifetime observed in Fig. 3(d) for the $\mathrm{Al}_{2} \mathrm{O}_{3}$-coated cell is partially associated to the increase of the charge-transfer resistance for the coated electrode, as shown in Fig. 3(b). In addition, the lifetime increase in the coated cell is also partly due to the increase of the capacitance that is observed in Fig. 3 (c). This is in contrast to the previous result ${ }^{11}$ where a small decrease of electron accumulation for alumina-coated films was obtained by chronoamperometry for unsensitized films in the dark. This different behavior may be attributed to the different experimental conditions, as the previous results were obtained recording the fast charging component of potential steps while here we report the quasiequilibrium capacitance at low frequencies. In the present context, it is important to remark that the charge-transfer resistance increases even in the presence of larger electron density associated to the increase of capacitance. Hence the combination of both effects of the coating layer, larger extent of electron accumulation, and decreased charge transfer to acceptors impacts positively on the solar cell efficiency. In conclusion the resistance and lifetime results of Fig. 3 indicate that the coating produces a reduction of the interfacial charge-transfer kinetics to the redox couple via the conduction-band mechanism.

It is important also to probe the region of lower Fermi levels, as here the charge transfer should be predominantly dominated by the trap/surface states in the band gap of $\mathrm{TiO}_{2}$. As mentioned, the impedance technique could not provide information on this region. We therefore turn now to consideration of the results of open-circuit voltage decay technique, ${ }^{17}$ which is able to monitor the kinetics of recombination in a DSSC in the domain of low photovoltages. The electron lifetime determined by this OCVD technique, shown in Fig. 3(d) as solid lines, shows an excellent agreement of the lifetime determined independently by EIS in the high potential domain where they overlap. While the agreement was conjectured previously, ${ }^{17}$ it is nonetheless remarkable because the two techniques utilize quite different methods. The model ${ }^{17}$ published for describing OCVD predicts an exponential dependence of the lifetime, i.e., a linear shape in the log/linear plot shown in Fig. 3(d). This model assumes trapping and release of electrons, and interfacial charge transfer proceeds only through the conduction band and should apply correctly in the region where the Fermi level is relatively close to the conduction band. These additional measurements reinforce the conclusion obtained above by the EIS data that the alumina barrier reduces by a factor $\approx 3$ the rate of charge recombination from the $\mathrm{TiO}_{2}$ conduction band to the oxidized electrolyte. It is apparent, however, that for the uncoated film, the OCVD lifetime plotted in Fig. 3(d) deviates significantly from an exponential dependence upon device voltage at low potentials. We present elsewhere a detailed analysis of such behavior, ${ }^{29}$ which attributes this 
curved region at lower values of potential to interfacial charge transfer from electrode surface states to the distributed energy levels of the acceptor species. In contrast to the uncoated film, for the $\mathrm{TiO}_{2} / \mathrm{Al}_{2} \mathrm{O}_{3}$ electrode, the OCVD line is almost straight with a slight curvature only below $-0.5 \mathrm{~V}$, implying that the transfer through conduction band is dominant up to very low values of the Fermi level in the $\mathrm{TiO}_{2}$ film. These results provide further evidence that the charge transfer from surface states has been strongly suppressed by the alumina coating, in agreement with the preceding conclusions by other techniques. We emphasize that the OCVD results relate to a DSSC in operating conditions (at open circuit), so that they provide a direct indication of the factors governing the higher efficiency of the solar cell obtained with the ultrathin barrier alumina coating.

\section{CONCLUSIONS}

Several techniques have been used to probe the kinetic effect of ultrathin alumina coating in DSSC. It is found that the barrier reduces the recombination of photoinjected electrons to both dye cations and the oxidized redox couple. It is proposed that this observed retardation can be attributed to primarily two effects: passivating almost completely surface trap states in $\mathrm{TiO}_{2}$ that are able to inject electrons to acceptor species and slowing down by a factor of 3-4 the rate of interfacial charge transfer from conduction-band states.

\section{ACKNOWLEDGMENTS}

Support of this work by Fundació Caixa Castelló under project P1B99-04 is acknowledged. One of the authors (J.D.) gratefully acknowledges the financial support from the EPSRC and the EU (Contract No. ENK6-CT-2001-00560 Nanomax). Another author (E.P.) is very grateful for the support of a Marie Curie Fellowship Contract No. HPMF-CT2002-01744.

${ }^{1}$ B. O'Regan, and M. Gratzel, Nature (London) 353, 737 (1991).

${ }^{2}$ J. Bisquert, D. Cahen, G. Hodes, S. Rühle, and A. Zaban, J. Phys. Chem.
B 108, 8106 (2004)

${ }^{3}$ C. J. Brabec, N. S. Saricifti, and J. C. Hummelen, Adv. Mater. (Weinheim, Ger.) 11, 15 (2001).

${ }^{4}$ H. Reiss, J. Phys. Chem. 89, 3783 (1985).

${ }^{5}$ L. M. Peter, E. A. Ponomarev, G. Franco, and N. J. Shaw, Electrochim. Acta 45, 549 (1999).

${ }^{6}$ G. R. R. A. Kumara, K. Tennakone, V. P. S. Perera, A. Konno, S. Kaneko, and M. Okuya, J. Phys. D 41, 868 (2001).

${ }^{7}$ A. Zaban, S. G. Chen, S. Chappel, and B. A. Gregg, Chem. Commun. (Cambridge) 22, 2231 (2000).

${ }^{8}$ S. Chappel, S. G. Chen, and A. Zaban, Langmuir 18, 3336 (2002).

${ }^{9}$ Y. Diamant, S. G. Chen, O. Melamed, and A. Zaban, J. Phys. Chem. B 107, 1977 (2003).

${ }^{10}$ E. Palomares, J. N. Clifford, S. A. Haque, T. Lutz, and J. R. Durrant, Chem. Commun. (Cambridge) 14, 1464 (2002).

${ }^{11}$ E. Palomares, J. N. Clifford, S. A. Haque, T. Lutz, and J. R. Durrant, J. Am. Chem. Soc. 125, 475 (2003).

${ }^{12}$ F. Lenzmann, M. Nanu, O. Kijatkina, and A. Belaidi, Thin Solid Films 451-452, 639 (2004).

${ }^{13}$ X. T. Zhang, H.-W. Liu, T. Taguchi, R.-B. Meng, O. Sato, and A. Fujishima, Sol. Energy Mater. Sol. Cells 81, 197 (2004).

${ }^{14}$ J. Bisquert, J. Phys. Chem. B 106, 325 (2002).

${ }^{15}$ F. Fabregat-Santiago, G. Garcia-Belmonte, J. Bisquert, A. Zaban, and P. Salvador, J. Phys. Chem. B 106, 334 (2002).

${ }^{16}$ R. Kern, R. Sastrawan, J. Ferber, R. Stangl, and J. Luther, Electrochim. Acta 47, 4213 (2002).

${ }^{17}$ A. Zaban, M. Greenshtein, and J. Bisquert, Chem. Phys. Chem. 4, 859 (2004).

${ }^{18}$ J. Bisquert and V. S. Vikhrenko, J. Phys. Chem. B 108, 2313 (2004).

${ }^{19}$ J. Bisquert, Phys. Chem. Chem. Phys. 5, 5360 (2003).

${ }^{20}$ A. Rose, Concepts in Photoconductivity and Allied Problems (Interscience, New York, 1963).

${ }^{21}$ S. A. Haque, Y. Tachibana, D. R. Klug, and J. R. Durrant, J. Phys. Chem. B 102, 1745 (1998).

${ }^{22}$ J. Nelson,S. A. Haque, D. R. Klug, and J. R. Durrant, Phys. Rev. B 63, 205321 (2001).

${ }^{23}$ S. A. Haque, Y. Tachibana, R. L. Willis, J. E. Moser, M. Grätzel, D. R. Klug, and J. R. Durrant, J. Phys. Chem. B 104, 538 (2000).

${ }^{24}$ J. Bisquert, A. Zaban, and P. Salvador, J. Phys. Chem. B 106, 8774 (2002).

${ }^{25}$ P. Salvador, M. González-Hidalgo, A. Zaban, and J. Bisquert (unpublished).

${ }^{26}$ W. D. Clark and N. Sutin, J. Am. Chem. Soc. 99, 4676 (1977).

${ }^{27}$ D. Kunciasuskas, M. S. Freund, H. B. Gray, J. R. Winkler, and N. Lewis, J. Phys. Chem. B 105, 392 (2001).

${ }^{28}$ T. Dittrich et al., Appl. Surf. Sci. (in press).

${ }^{29}$ J. Bisquert, A. Zaban, M. Greenshtein, and I. Mora-Seró, J. Am. Chem. Soc. 126, 13550 (2004). 